ORIGINAL

\title{
Non-invasive Measurement of Skin Autofluorescence as a Beneficial Surrogate Marker for Atherosclerosis in Patients with Type 2 Diabetes
}

\author{
Jin Temma ${ }^{1}$, Munehide Matsuhisa², Toru Horie ${ }^{3}$, Akio Kuroda², Hiroyasu Mori², Motoyuki Tamaki², Itsuro Endo', \\ Ken-ichi Aihara ${ }^{1}$, Masahiro Abe ${ }^{1}$, and Toshio Matsumoto ${ }^{4}$
}

\begin{abstract}
${ }^{1}$ Department of Hematology, Endocrinology \& Metabolism, Institute of Biomedical Sciences, Tokushima University Graduate School, Tokushima, Japan, ${ }^{2}$ Diabetes Therapeutics and Research Center, Tokushima University, Tokushima, Japan, ${ }^{3}$ Tenma Hospital, Tokushima, Japan, ${ }^{4}$ Fujii Memorial Institute of Medical Sciences, Tokushima University, Tokushima, Japan
\end{abstract}

\begin{abstract}
Advanced glycation end-products (AGEs) are thought to play a major role in the pathogenesis of diabetic vascular complications. Skin autofluorescence (AF) was recently reported to represent tissue AGEs accumulation with a non-invasive method. The aim of the present study was to evaluate association between AF value and diabetic vascular complications, such as retinopathy, nephropathy and cervical atherosclerosis using the carotid intima-media thickness (IMT), an established marker of cardiovascular disease in patients with type 2 diabetes. A total of 68 patients with type 2 diabetes were enrolled in a cross-sectional manner. AGEs accumulation was measured with AF reader. Clinical parameters were collected at the time of AF and IMT measurement. Max-IMT was correlated with age and AF $(r=0.407, p=0.001)$, but not with HbA1c, GA, and pentosidine. Also, AF was not correlated with HbA1c, GA and pentosidine, but was correlated with age $(\mathbf{r}=0.560, p<0.001)$, duration of diabetes $(r=0.256, p<0.05)$. Multivariate regression analysis revealed that $A F$, but not age, was an independent determinant of max-IMT. In conclusion, AF might be a beneficial surrogate marker for evaluating carotid atherosclerosis in patients with type 2 diabetes non-invasively. J. Med. Invest. 62 : 126-129, August, 2015
\end{abstract}

Keywords : skin autofluorescence, intima-media thickness, type 2 diabetes, atherosclerosis

\section{INTRODUCTION}

Diabetic vascular complications shorten the lifespan and deteriorated the quality of life in patients with type 2 diabetes. To prevent these complications, clinical application of useful marker for microvascular and macro-vascular is necessary.

Non-enzymatic glycation of proteins is a series of complex and sequential reactions collectively called the Maillard reaction. Earlystage reactions lead to the formation of the early glycation adducts, and later-stage reactions subsequently form advanced glycation end-products (AGEs). Increased formation and accumulation of AGEs could be a major pathogenic mechanism in developing atherosclerosis in type 2 diabetes (1). The accumulation of AGEs on tissue proteins has been implicated as a contributing factor in the progression of diabetes complications (2-6).

Tissue AGEs accumulation may reflect the cumulative effect of hyperglycemia over many years. Therefore, tissue AGEs accumulation must represent the existing tissue damage resulting from various known risk factors under diabetic condition (7). Skin autofluorescence $(\mathrm{AF})$ has a strong correlation with the specific AGEs content in skin biopsies, as shown in multiple validation studies (8-10). Simple quantitation of accumulated AGEs in the tissue could provide a tool for assessing tissue injury and the risk of long-term complications (8). Non-invasive measurement of AF has been proposed as a marker of accumulation of AGEs in the skin (11). AF is increased in patients with type 2 diabetes in conjunction with macrovascular disease (9). Increased $\mathrm{AF}$ was reported to predict

Received for publication December 15, 2014 ; accepted December 26, 2014.

Address correspondence and reprint requests to Munehide Matsuhisa 3-18-15, Kuramoto-cho, Tokushima 770-8503, Japan and Fax : +81-88633-7589. cardiovascular events independent of conventional risk factors (12).

Carotid intima-media thickness (IMT) is an especially useful marker of the progression of atherosclerosis throughout the body, and is an excellent predictor of cardiovascular events. As a simple and non-invasive procedure, measurement of carotid IMT is one of the most appropriate screening methods to specify high-risk individuals in subjects with and without diabetes (13). It is reported that elevated AF is associated with IMT in non-diabetic subjects (14). So far, there are no reports describing about the relation between AF, IMT, and other clinical characteristics in patients with type 2 diabetes.

The objective of this study is to investigate the correlation between AF, IMT, and other clinical characteristics in patients with type 2 diabetes in a cross-sectional manner.

\section{MATERIALS AND METHODS}

This cross-sectional study was performed in Tenma Hospital, Tokushima, Japan, from May to October in 2014. The present study was carried out in accordance with the Declaration of Helsinki and with approval from the ethical committee of Tenma Hospital. The written informed consent was obtained from each patient. The inclusion criteria were patients with type 2 diabetes who were below 80 years old. The exclusion criteria was patients with moderate to end-stage renal failure $\left(\mathrm{eGFR}<30 \mathrm{ml} / \mathrm{min} / 1.73 \mathrm{~m}^{2}\right.$ ), anemia (hemoglobin $<10 \mathrm{~g} / \mathrm{dl}$ ), and extremely sunburn. A total of 68 patients who met the inclusion and exclusion criteria were consecutively selected in this study. We also excluded individuals with unstable retinopathy, pregnancy or using steroids. All patients were studied after an overnight fast. BMI was calculated as weight divided by height squared (in $\mathrm{kg} \cdot \mathrm{m}^{-2}$ ). Waist circumstance was measured between the $10^{\text {th }}$ rib and the iliac crest. 
HbA1c was measured with high-performance liquid chromatography method. $\mathrm{HbA}_{1 \mathrm{c}}$ was expressed as a National Glycohemoglobin Standardization Program (NGSP) equivalent value. Glycated albumin was determined by an enzymatic method using a liquid chemistry system (Lucica GA-L method, Asahi Kasei Corp., Tokyo). For an assessment for urine albumin/creatinine ratio (UACR), urine albumin and creatinine were measured from a single random voided urine sample. Urine albumin levels were measured by immunoturbimetric assay (ALBT2, Roche, Basel, Switzerland). Urine creatinine levels were measured using the Jaffe method (CREJ2, Roche). Serum creatinine and lipid levels [total, high-density lipoprotein (HDL), and low-density lipoprotein (LDL) cholesterol and triglycerides] were measured immediately using standard methods. Plasma pentosidine concentrations were measured using a commercially available competitive enzyme-linked immunosorbent assay (ELISA) (FSK pentosidine ELISA kit ; Fushimi Pharmaceutical, Kagawa, Japan) ; the samples were stored at $-80^{\circ} \mathrm{C}$ until analysis. Micro-albuminuria in the stage 2 of diabetic nephropathy and macro-albuminuria in the stage 3 of diabetic nephropathy were defined as the urinary albumin/creatinine excretion ratio more than 30 and $300 \mathrm{mg} / \mathrm{gCr}$, respectively. Retinopathy was diagnosed as non-diabetic retinopathy (NDR), simple diabetic retinopathy (SDR), and pre-proliferative retinopathy (PPDR) by ophthalmologists.

\section{Measurement of $A F$}

$\mathrm{AF}$ was measured with the advanced glycation end product (AGE) Reader TM (DiagnOptics Technologies BV, Groningen, the Netherlands). The AGE Reader is a desktop device that uses the characteristic fluorescent properties of some advanced glycation end-products to estimate the level of advanced glycation endproduct accumulation in the skin. Technical and optical details of this non-invasive method have been described more extensively elsewhere (11). In short, the AGE Reader illuminates a skin surface of $4 \mathrm{~cm}^{2}$, guarded against surrounding light, with an excitation light source with a peak excitation of $370 \mathrm{~nm}$. This wavelength is in the UVA spectrum. Emission light in the wavelength range of 420-600 $\mathrm{nm}$ (fluorescence) and excitation light that is reflected by the skin with a wavelength range of $300-420 \mathrm{~nm}$ from the skin is measured with a spectrometer. Skin autofluorescence was determined from the ratio between the emission light and the reflected excitation light, using the AGE Reader software, version 2.2. AF was measured at room temperature while patients were at rest in a seated position. The forearm was positioned on the top of device in the usual manner, as described by the manufacturer. Measurements were not specifically performed in a fasting state. For each skin autofluorescence value, three consecutive measurements were carried out at three different skin sites of the same forearm, within a total test period of approximately $2 \mathrm{~min}$. The mean of these three consecutive measurements was used in the analyses. Skin pigmentation influences the measurement of skin autofluorescence and its influence has been extensively studied and reported earlier (15).

\section{Measurement of max-IMT}

IMT was measured by one laboratory technician in accordance with a previous report (13). In brief, using B-mode ultrasonography, the carotid arteries are scanned in transverse sections from the origins of the common carotid arteries, carotid sinuses, internal carotid arteries and external carotid arteries, and then examined for carotid lesions in longitudinal sections at different angles. High-resolution ultrasound images with a range resolution of $0.1 \mathrm{~mm}$ can be obtained when carotid ultrasonography is carried out using a linear probe with a center frequency of $8.9 \mathrm{MHz}$ (GM-72P00A, Panasonic Healthcare Co. Japan) (13). Among IMT values, max-IMT is superior to mean-IMT in distinguishing patients with coronary stenosis $(13,16,17)$, thus max-IMT was measured in this study.

\section{Statistical Analysis}

Demographic data are presented as means \pm standard deviation (SD). Between-group differences of the average were compared using Mann-Whitney U test for non-parametric data. Categorical variables were compared using $\chi^{2}$-test. Spearman's analysis was done for non-parametric variables. Stepwise regression analysis was indicated to AF and age. For statistical analyses were performed with SPSS version 22.0 software. For all tests, $p<0.05$ was considered statistically significant.

\section{RESULTS}

The data was successfully measured in 61 patients among 68 patients. The characteristics of these study participants were shown in Table 1. Treatment for diabetes was diet alone in 9 patients, oral hypoglycemic agents (OHA) in 40 patients, and insulin with and without OHA in 7 and 5 patients. Thirty three patients and 21 patients were treated by anti-hypertensive agents and lipid-lowering agents, respectively. SDR and PPDR were observed in 5 patients, respectively. Fourteen patients were in the stage 2 and three were in the stage 3 of diabetic nephropathy.

Because the data are not normally distributed, Spearmann's nonparametric test was applied to the statistical analyses. The correlations between max-IMT, AF and clinical characteristics were shown in Table 2 . AF was significantly correlated with age $(r=$ $0.560, p<0.0001)$, duration of diabetes $(r=0.256, p<0.05)$, serum creatinine $(r=0.264, p<0.05)$, and max-IMT $(r=0.407, p<0.001$, Figure 1). Max-IMT also tended to be correlated with age $(r=$ $0.249, \mathrm{p}=0.053$ ). Multivariate regression analysis using $\mathrm{AF}$ and age for the dependent factor revealed that $\mathrm{AF}$ was only an independent determinant of $\max$-IMT $(R=0.425, \beta=0.425, \mathrm{p}<0.01)$.

In addition, $\mathrm{AF}$ was significantly higher in patients with diabetic

Table 1. Patients' Characteristics

\begin{tabular}{lc}
\hline Number of subjects & 61 \\
Gender (male/female) & $38 / 23$ \\
Age (years) & $66.6 \pm 9.2$ \\
Duration of diabetes (years) & $10.4 \pm 7.3$ \\
BMI(kg/m²) & $25.5 \pm 4.6$ \\
Systolic blood pressure (mmHg) & $130.7 \pm 15.5$ \\
Diastolic blood pressure (mmHg) & $70.9 \pm 9.3$ \\
HbA1c (\%) & $7.0 \pm 0.8$ \\
Glycated albumin (\%) & $17.9 \pm 2.7$ \\
Pentosidine ( $\mu \mathrm{l} / \mathrm{mL})$ & $0.032 \pm 0.017$ \\
Creatinine (mg/dL) & $0.72 \pm 0.16$ \\
eGFR (ml/min/1.73 m2) & $78.9 \pm 22.9$ \\
UAER (mg/gCr) & $55.1 \pm 161.4$ \\
Total cholesterol (mg/dL) & $186.8 \pm 34.0$ \\
Triglyceride (mg/dL) & $145.7 \pm 73.7$ \\
HDL-cholesterol (mg/dL) & $49.2 \pm 13.3$ \\
LDL-cholesterol (mg/dL) & $108.5 \pm 30.2$ \\
AF & $2.50 \pm 0.50$ \\
Max-IMT (mm) & $1.64 \pm 0.75$ \\
Retinopathy (NDR/SDR/PPDR) & $(51 / 5 / 5)$ \\
Nephropathy (stage1/2/3) & $(44 / 14 / 3)$ \\
\hline
\end{tabular}

BMI ; body mass index, eGFR; estimated glomerular filtration rate, UAER ; urinary albumin excretion rate, AF ; autofluorescence, NDR ; nondiabetic retinopathy, SDR; simple diabetic retinopathy, PPDR; preproliferative retinopathy, Max-IMT ; maxim intima-media thickness in the carotid artery. 
Table 2. The correlation between AF, IMT and clinical characteristics (Spearmann's nonparametric test)

\begin{tabular}{|l|r|r|r|r|}
\hline \multirow{2}{*}{ Age } & \multicolumn{3}{|c|}{ Spearmann's non-parametric test } \\
\cline { 2 - 5 } & \multicolumn{2}{|c|}{ Max-IMT } & \multicolumn{2}{c|}{ AF } \\
\hline Duration of diabetes & \multicolumn{1}{c|}{$\mathrm{r}$} & $\mathrm{p}$ value & \multicolumn{1}{c|}{$\mathrm{r}$} & $\mathrm{p}$ value \\
\hline Waist Circumference & 0.162 & 0.053 & 0.560 & 0.000 \\
\hline BMI & 0.078 & 0.553 & 0.045 & 0.730 \\
\hline HbA1c & -0.098 & 0.451 & -0.074 & 0.569 \\
\hline Glycated Albumin & 0.090 & 0.493 & 0.092 & 0.479 \\
\hline UAER & 0.123 & 0.344 & -0.022 & 0.866 \\
\hline Serum creatinine & -0.085 & 0.512 & 0.048 & 0.715 \\
\hline Pentosidine & 0.226 & 0.079 & 0.264 & 0.040 \\
\hline eGFR & -0.086 & 0.511 & 0.041 & 0.753 \\
\hline Max-IMT & -0.021 & 0.870 & -0.226 & 0.080 \\
\hline Systolic blood pressure & NA & NA & 0.407 & 0.001 \\
\hline Diastolic blood pressure & 0.015 & 0.910 & 0.149 & 0.252 \\
\hline Total cholesterol & -0.132 & 0.312 & -0.145 & 0.264 \\
\hline Triglyceride & -0.059 & 0.650 & -0.194 & 0.135 \\
\hline HDL-cholesterol & -0.205 & 0.114 & 0.038 & 0.775 \\
\hline LDL-cholesterol & 0.077 & 0.553 & -0.219 & 0.091 \\
\hline BMI bory & 0.220 & 0.881 & -0.201 & 0.110 \\
\hline
\end{tabular}

BMI ; body mass index, eGFR; estimated glomerular filtration rate, UAER ; urinary albumin excretion rate, AF ; autofluorescence, Max-IMT ; maxim intima-media thickness in the carotid artery.

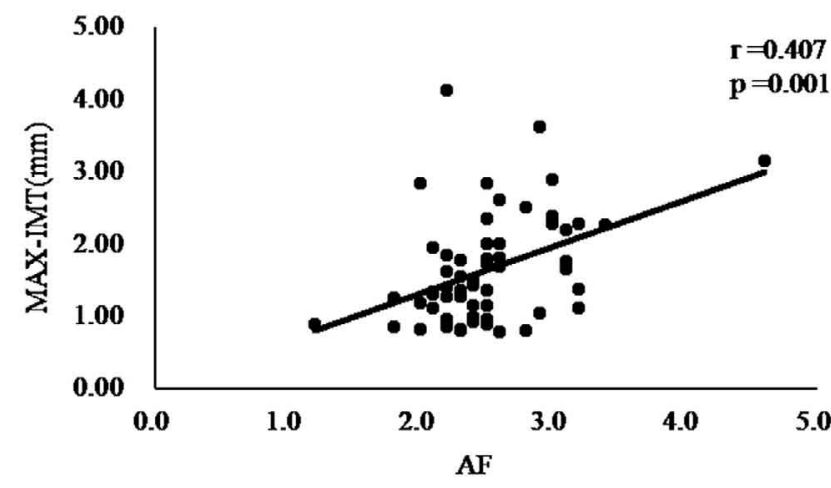

Figure 1. The correlation between MAX-IMT and AF

retinopathy (SDR or PPDR) compared to the patients without diabetic retinopathy $(3.03 \pm 0.67$ vs $2.41 \pm 0.40, \mathrm{p}<0.01)$. There was no significant difference of $\mathrm{AF}$ between with and without diabetic nephropathy ( $2.46 \pm 0.43$ vs $2.63 \pm 0.63$, n.s.).

\section{DISCUSSION}

The present study showed that subcutaneous accumulation of AGE was well correlated with the degree of max-IMT of the carotid artery. On the other hand, HbA1c, glycated albumin, and pentosidine were not correlated with max-IMT.

Increased AGE-RAGE signal was a major cause of atherosclerosis in patients with diabetes. Since pathological change of atherosclerosis progresses gradually, short duration of glycated protein, such as HbA1c and glycated albumin, were unable to reflect it. Measurement of tissue AGEs may be preferable over plasma measurement, since long-lived proteins accumulate in the tissues in which chronic complications develop (18). Thus, blood and urine
AGEs do not necessarily reflect their tissue levels (19). Indeed, pentosidine, a fluorescent advanced glycation product, was also reported to correlate well with $\mathrm{AF}(8)$, however, there are no correlation between serum pentosidine and max-IMT and $\mathrm{AF}$. We also failed to find the correlation between max-IMT and pentosidine in this study.

Previous reports have shown that increased accumulation of AGE in tissue was a major cause of micro-vascular complications (2-6). In the present study, subcutaneous accumulation of AGEs was correlated with the presence of diabetic retinopathy, but not diabetic nephropathy. Because the development and progression of kidney injury in diabetic patients were not only due to chronic hyperglycemia, but also hypertension, smoking, obesity, and use of renin-angiotensin inhibitor, we failed to observe the correlation between $\mathrm{AF}$ and nephropathy, as reported previously $(20,21)$. As mentioned, renal failure is not only because of hyperglycemia, we excluded patients with end-stage renal failure in this study. In addition, renal failure itself is known to increase AGEs formation.

There are some limitations in our study. The number of the patients seems small to conclude in this cross-sectional study, however, it was enough to find the correlation between the AF and max-IMT. We usually use mean-IMT and max-IMT values to evaluate atherosclerosis in patients with type 2 diabetes. The IMT value is only evaluated with max-IMT in this study. We experienced that max-IMT represents better than mean-IMT in evaluation of the atherosclerosis in patients with type 2 diabetes $(16,17)$.

In conclusion, non-invasive measurement of $\mathrm{AF}$ might be a good surrogate marker for evaluating atherosclerosis and micro-angiopathy in patients with type 2 diabetes.

\section{CONFLICT OF INTEREST}

The authors declare that they have no conflicts of interest associated with the present study.

\section{ACKNOWLEDGMENTS}

We acknowledge Kazumi Hata for excellent secretarial work.

\section{REFERENCES}

1. Goldin A, Beckman JA, Schmidt AM, Creager MA : Advanced glycation end products. Sparking the development of diabetic vascular injury. Circulation 114 : 597-605, 2006

2. Beisswenger PJ, Makita Z, Curphey TJ, Moore LL, Jean S, Brinck-Johnsen T, Bucala R, Vlassara $\mathrm{H}$ : Formation of immunochemical advanced glycosylation end products precedes and correlates with early manifestations of renal and retinal disease in diabetes. Diabetes $44: 824-829,1995$

3. Dyer DG, Dunn JA, Thorpe SR, Bailie KE, Lyons TJ, McCance DR, Baynes JW : Accumulation of Maillard reaction products in skin collagen in diabetes and aging. J Clin Invest 91 : 24632469, 1993

4. McCance DR, Dyer DG, Dunn JA, Bailie KE, Thorpe SR, Baynes JW, Lyons TJ : Maillard reaction products and their relation to complications in insulin-dependent diabetes mellitus. J Clin Invest $91: 2470-2478,1993$

5. Monnier VM, Vishwanath V, Frank KE, Elmets CA, Dauchot P, Kohn RR: Relation between complications of type I diabetes mellitus and collagen-linked fluorescence. N Engl J Med $314:$ 403-408, 1986

6. Genuth S, Sun W, Cleary P, Sell DR, Dahms W, Malone J, Sivitz W, Monnier VM : Glycation and carboxymethyllysine 
levels in skin collagen predict the risk of future 10-year progression of diabetic retinopathy and nephropathy in the diabetes control and complications trial and epidemiology of diabetes interventions and complications participants with type 1 diabetes. Diabetes 54 : 3103-3111, 2005

7. Meerwaldt R, Lutgers HL, Links TP, Graaff R, Baynes JW, Gans RO, Smit AJ : Skin autofluorescence is a strong predictor of cardiac mortality in diabetes. Diabetes Care 30 : 107 112,2007

8. Meerwaldt R, Links T, Graaff R, Thorpe SR, Baynes JW, Hartog J, Gans R, Smit A : Simple noninvasive measurement of skin autofluorescence. Ann N Y Acad Sci 1043 : 290-298, 2005

9. Lutgers HL, Graaff R, Links TP, Ubink-Veltmaat LJ, Bilo HJ, Gans RO, Smit AJ : Skin autofluorescence as a noninvasive marker of vascular damage in patients with type 2 diabetes. Diabetes Care 29 : 2654-2659, 2006

10. Gerrits EG, Lutgers HL, Kleefstra N, Graaff R, Groenier KH, Smit AJ, Gans RO, Bilo HJ : Skin autofluorescence : a tool to identify type 2 diabetic patients at risk for developing microvascular complications. Diabetes Care 31 : 517-521, 2008

11. Meerwaldt R, Graaff R, Oomen PH, Links TP, Jager JJ, Alderson NL, Thorpe SR, Baynes JW, Gans RO, Smit AJ : Simple non-invasive assessment of advanced glycation endproduct accumulation. Diabetologia $47: 1324-1330,2004$

12. Lutgers HL, Gerrits EG, Graaff R, Links TP, Sluiter WJ, Gans RO, Bilo HJ, Smit AJ : Skin autofluorescence provides additional information to the UK Prospective Diabetes Study (UKPDS) risk score for the estimation of cardiovascular prognosis in type 2 diabetes mellitus. Diabetologia 52 : 789-797, 2009

13. Katakami N, Kaneto H, Shimomura I : Carotid ultrasonography : A potent tool for better clinical practice in diagnosis of atherosclerosis in diabetic patients. J Diabetes Investig $5: 3-13$, 2014

14. Lutgers HL, Graaff R, de Vries R, Smit AJ, Dullaart RP : Carotid artery intima media thickness associates with skin autofluoresence in non-diabetic subjects without clinically manifest cardiovascular disease. Eur J Clin Invest $40: 812-817,2010$

15. Koetsier M, Nur E, Chunmao H, Lutgers HL, Links TP, Smit AJ, Rakhorst G, Graaff R: Skin color independent assessment of aging using skin autofluorescence. Opt Express 18 : 1441614429, 2010

16. Kasami R, Kaneto H, Katakami N, Sumitsuji S, Yamasaki K, Kuroda T, Tachibana K, Yasuda T, Kuroda A, Matsuoka TA, Matsuhisa M, Shimomura I : Relationship between carotid intima-media thickness and the presence and extent of coronary stenosis in type 2 diabetic patients with carotid atherosclerosis but without history of coronary artery disease. Diabetes Care 34 : 468-470, 2011

17. Irie Y, Katakami N, Kaneto H, Kasami R, Sumitsuji S, Yamasaki K, Tachibana K, Kuroda T, Sakamoto K, Umayahara Y, Ueda Y, Kosugi K, Shimomura I : Maximum carotid intima-media thickness improves the prediction ability of coronary artery stenosis in type 2 diabetic patients without history of coronary artery disease. Atherosclerosis $221: 438-444,2012$

18. Meerwaldt R, Links T, Zeebregts C, Tio R, Hillebrands JL, Smit A : The clinical relevance of assessing advanced glycation end-products accumulation in diabetes. Cardiovasc Diabetol $7: 29,2008$

19. Hricik DE, Wu YC, Schulak JA, Friedlander MA : Disparate changes in plasma and tissue pentosidine levels after kidney and kidney-pancreas transplantation. Clin Transplant 10 : 568-573, 1996

20. Katayama S, Moriya T, Tanaka S, Yajima Y, Sone H, limuro S, Ohashi Y, Akanuma Y, Yamada $\mathrm{N}$ : Low transition rate from normo- and low microalbuminuria to proteinuria in Japanese type 2 diabetic individuals : the Japan Diabetes Complications Study (JDCS). Diabetologia $54: 1025-1031,2011$

21. Araki S, Haneda M, Koya D, Hidaka H, Sugimoto T, Isono M, Isshiki K, Chin-Kanasaki M, Uzu T, Kashiwagi A : Reduction in microalbuminuria as an integrated indicator for renal and cardiovascular risk reduction in patients with type 2 diabetes. Diabetes 56 : 1727-1730, 2007 